RR 343

CRREL

Research Report 343

SIMULTANEOUS MEASUREMENT OF

LASER EXTINCTION IN WARM FOG AT

WAVELENGTHS OF $0.6328,1.15$ AND 10.6 MICRONS

Richard H. Munis and Allan J. Delaney

October 1975

\author{
Prepared for \\ ADVANCED RESEARCH PROJECTS AGENCY \\ By \\ CORPS OF ENGINEERS, U.S. ARMY \\ COLD REGIONS RESEARCH AND ENGINEERING LABORATORY \\ HANOVER, NEW HAMPSHIRE
}


The findings in this report are not to be construed as an official Department of the Army position unless so designated by other authorized documents. 


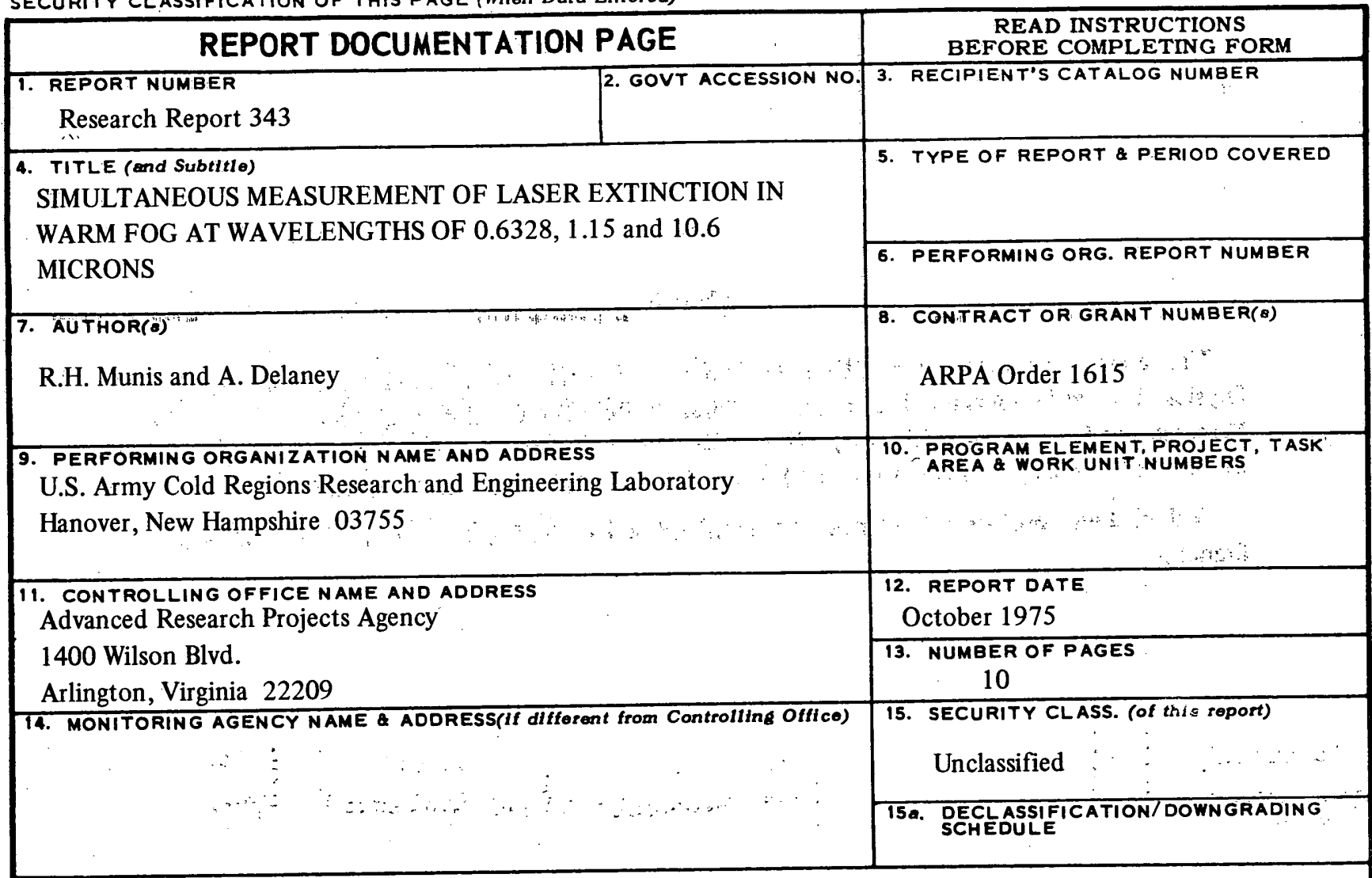

\section{DISTRIBUTION STATEMENT (of this Roport)}

Approved for public release; distribution unlimited.

17. DISTRIBUTION STATEMENT (of the abstract onterod in Block 20, if difforent from Roport)

18. SUPPLEMENTARY NOTES

19. KEY WORDS (Continue on reverae aide If necesaery and Idontify by block number)

Absorptivity

Laser beams

Optical radar

Surface effect vehicles

Visibility

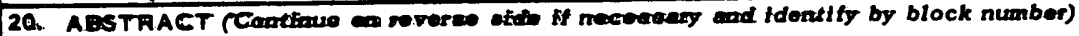

Simultaneous laser extinction measurements were made in warm fog at wavelengths of $0.6238,1.15$ and $10.6 \mu$. The warm fog was generated in a $4-\mathrm{m}^{3}$ environmental chamber. Particle sampling was carried out simultaneously with the laser measurements using an impactor. Using the same size distribution in each case the theoretical extinction coefficients were calculated and compared with the experimental coefficients. Results obtained during this experiment and a previous one indicate that propagation at $1.15 \mu$ is adversely affected by the presence of atmospheric water vapor. Experimental data obtained simultaneously at 0.6328 and $10.6 \mu$ indicate that virtually no difference exists between the extinction coefficients at these two wavelengths for moderate particle concentrations while at much larger concentrations $\alpha_{\mathbf{0 . 6 3 2 8}}$ increases indefinitely while $\alpha_{10.6}$ levels off at 0.2 . 


\section{PREFACE}

This report was prepared by Dr. Richard H. Munis, Research Physicist, and Allan Delaney, Physical Science Technician, Physical Sciences Branch, Research Division, U.S. Army Cold Regions Research and Engineering Laboratory. The work was performed as part of the Arctic Surface Effect Vehicle program of the Advanced Research Projects Agency under ARPA Order 1615.

Technical review of the manuscript was performed by Dr. Yin-Chao Yen, Chief, Physical Sciences Branch. 


\section{CONTENTS}

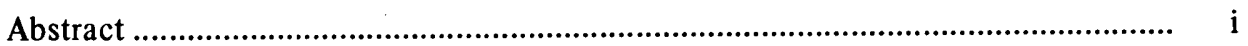

Preface

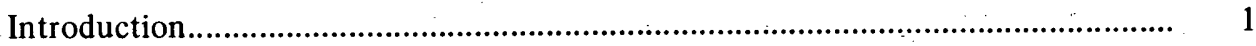

Experimental procedure …..................................................................................... 1

Experimental and theoretical equations …............................................................ 1

Results and discussion ..................................................................................... 3

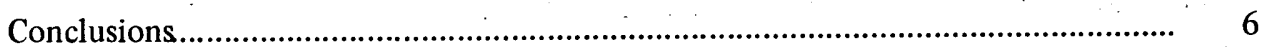

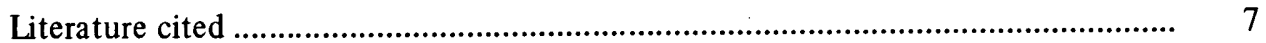

\section{ILLUSTRATIONS}

Figure

1. $Q_{\text {ext }}$ vs particle radius at three wavelengths

2. Particle concentration vs diameter at $-4^{\circ} \mathrm{C}$

3. Theoretical extinction coefficient at $10.6 \mu$ vs theoretical coefficient at

$0.6328 \mu$

4. Experimental extinction coefficient at $10.6 \mu$ vs experimental coefficient at $0.6328 \mu$

\section{TABLES}

Table

I. Experimental extinction coefficients of warm fog at $-4^{\circ} \mathrm{C}$

II. Transmission values and corresponding optical depths at 0.6328 and $10.6 \mu \ldots$ 


\title{
SIMULTANEOUS MEASUREMENT OF LASER EXTINCTION IN WARM FOG AT WAVELENGTHS OF 0.6328, 1.15 and 10.6 MICRONS
}

by

\author{
R.H. Munis and A. Delaney
}

\section{Introduction}

A previous paper ${ }^{3}$ reported on the theoretical and experimental extinction coefficients in ice fog at wavelengths of $0.6328,1.15$ and $3.39 \mu$. These data were required by the Advanced Research Projects Agency (ARPA) for the design of an obstacle avoidance system for a surface effect vehicle (SEV). Also part of their requirements was the need to determine the extinction coefficients through warm fog at $0.6328,1.15$ and $10.6 \mu$. Since a direct comparison of the extinction coefficient at these wavelengths was desired, it was decided to set up an experiment in the fog chamber.: whereby all three lasers could be operated simultaneously. This report presents theoretical and experimental data on simultaneous laser extinction measurements through warm fog at 0.6328 , 1.15 and $10.6 \mu$.

\section{Experimental procedure}

The simultaneous extinction measurements at $0.6328,1.15$ and $10.6 \mu$ were made in a $4-\mathrm{m}^{3}$ chamber whose temperature was maintained at $-4^{\circ} \mathrm{C}$ (a detailed description of this chamber is given in ref. 3). The three lasers were located so that, allowing for divergence of each beam at the detector, the distance between the 0.6328 - and $1.15-\mu$ beams was approximately one inch while the distance between these two beams and the $10.6-\mu$ beam was approximately two inches. The three beams had to be near each other so that they would propagate through a volume of hydrometeors that could be sampled conveniently with the hand-operated impactor described in ref. 3 . Thus the samples obtained with this instrument approximately described the hydrometeor size distributions which all three laser beams encountered simultaneously in the propagation path. These size distributions were then constructed using the technique discussed in ref. 3 to yield the so-called theoretical extinction coefficient. It should be pointed out that this extinction coefficient is not a true theoretically derived coefficient since the hydrometeor size distribution which is used in computing it is measured with the hand-operated impactor.

The procedure for measuring the transmission of the dissipating fog was identical with the technique discussed in ref. 3 , except that in this experiment three laser beams were propagating simultaneously through the fog. This enabled three simultaneous equations to be written describing the nature of the theoretical extinction coefficients and their dependency on the hydrometeor size distribution.

\section{Experimental and theoretical equations}

Following the discussion in ref. 3, we write the general equation to calculate the experimental extinction coefficient: 


$$
T[N(r), \lambda]=\mathrm{e}^{-\alpha_{\lambda}[N(r), \lambda] \ell}
$$

where $T[N(r), \lambda]=$ measured transmission, $\%$

$\alpha_{\lambda}=$ experimental extinction coefficient, $\mathrm{m}^{-1}$

$\ell=$ propagation path length, $\mathrm{m}$.

and $N(r)$ describes the hydrometeor size distribution which exists in the fog chamber at the instant of time in which the transmission measurements are taken.

Three simultaneous equations may be written for the theoretical extinction coefficient:

$$
\begin{gathered}
\alpha_{0.6328}=\Pi \sum_{r_{\min }}^{r_{\max }} N(r) r^{2} \Delta r Q_{0.6238}(m, X) \\
\alpha_{1.15}=\Pi \sum_{r_{\min }}^{r_{\max }} N(r) r^{2} \Delta r Q_{1.15}(m, X) \\
\alpha_{10.6}=\Pi \sum_{r_{\min }}^{r_{\max }} N(r) r^{2} \Delta r Q_{10.6}(m, X) .
\end{gathered}
$$

where, as before,

$$
\begin{aligned}
& \alpha_{\lambda}=\text { theoretical extinction coefficient, } \mathrm{m}^{-1} \\
& Q_{\text {ext }}=\text { van de Hulst's efficiency factor for total extinction } \\
& m=\text { complex index of refraction } \\
& X=\text { particle size parameter, } 2 \pi r / \lambda \\
& \text { and } N(\dot{r}) \text { is as discussed above. }
\end{aligned}
$$

It should be noted from eq $2-4$ that while the efficiency factor $Q_{\text {ext }}$ is different at each value of $\lambda$ the hydrometeor size distribution $N(r)$ remains essentially identical because of the nature of the experiment. Figure 1 shows the behavior of $Q_{\text {ext }}$ with particle radius for wavelengths of $0.6328,1.15$ and $10.6 \mu$. Note that the extinction efficiency of the complete particle spectrum is approximately

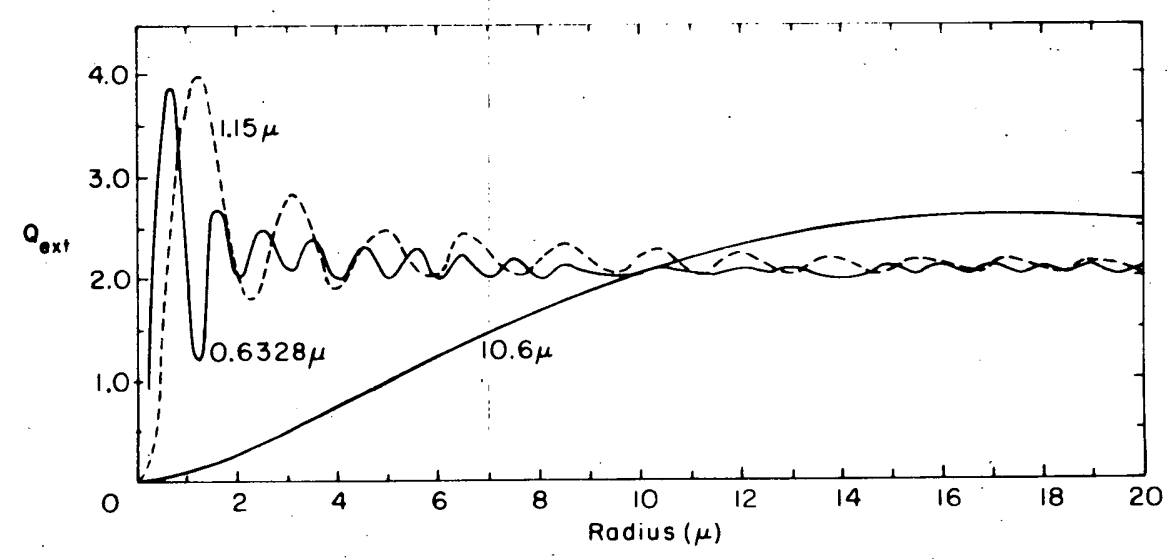

Figure 1. $Q_{\text {ext }}$ vs particle radius at three wavelengths. 
the same for 0.6328 and $1.15 \mu$, damping out eventually around a value of 2 . However, the situation at $10.6 \mu$ is quite different; the extinction efficiency is very low for small particles but grows considerably towards the larger end of the particle spectrum. From these graphs we could conclude that if the hydrometeor size spectra of a given experiment contained most of the particles in the range $0<r<10 \mu$ then the extinction coefficient at $10.6 \mu$ would be expected to be considerably lower than at 0.6328 or $1.15 \mu$. The results of our experiment indicate that the above proposition is true for $0.6328 \mu$ but not for $1.15 \mu$. More attention will be.given to this matter in the following section.

\section{Results and discussion}

Table I compares the transmission and extinction coefficients at $0.6328,1.15$ and $10.6 \mu$ for different hydrometeor concentrations. Figure 2 shows the hydrometeor spectra which resulted in the transmission values and extinction coefficients of Table $I$.

Table I. Experimental extinction coefficients of warm fog at $-4^{\circ} \mathrm{C}$.

\begin{tabular}{cccc}
$\begin{array}{c}\text { Particle } \\
\text { concentration } \\
\left(N / \mathrm{cm}^{3}\right)\end{array}$ & $\begin{array}{c}\text { Experimental } \\
\text { extinction } \\
\text { coefficient } \\
\left(\mathrm{m}^{-1}\right)\end{array}$ & $\begin{array}{c}\text { Transmission } \\
(\%)\end{array}$ & $\begin{array}{c}\text { Attenuation } \\
(\mathrm{db} / \mathrm{m})\end{array}$ \\
\hline
\end{tabular}

a. $0.6328 \mu$

$\begin{array}{rrrr}130 & 0.1450 & 56 & 0.0629 \\ 125 & 0.0729 & 75 & 0.0312 \\ 68 & 0.0377 & 86 & 0.0164 \\ 41 & 0.0181 & 93 & 0.0079 \\ 35 & 0.0527 & 81 & 0.0229 \\ 227 & 0.0181 & 93 & 0.0079 \\ 116 & 0.5101 & 13 & 0.2214 \\ 258 & 0.0527 & 81 & 0.0229 \\ 184 & 0.6648 & 7 & 0.2885 \\ & 0.2354 & 39 & 0.1022\end{array}$

b. $1.15 \mu$

$\begin{array}{rrrr} & 0.749 & 5 & 0.325 \\ 130 & 0.602 & 9 & 0.261 \\ 125 & 0.337 & 26 & 0.146 \\ 68 & 0.168 & 51 & 0.073 \\ 41 & 0.492 & 14 & 0.213 \\ 35 & 0.104 & 66 & 0.045 \\ 227 & 0.978 & 2 & 0.425 \\ 116 & 0.379 & 22 & 0.164 \\ 258 & 0.877 & 3 & 0.381 \\ 184 & 0.805 & 4 & 0.349\end{array}$

c. $10.6 \mu$

\begin{tabular}{rrrr} 
& 0.1362 & 58 & 0.0591 \\
130 & 0.0753 & 74 & 0.0327 \\
125 & 0.0291 & 89 & 0.0216 \\
68 & 0.0051 & 98 & 0.0022 \\
41 & 0.0496 & 82 & 0.0215 \\
35 & 0.0181 & 93 & 0.0079 \\
227 & 0.1941 & 46 & 0.0843 \\
116 & 0.0291 & 89 & 0.0216 \\
258 & 0.1783 & 49 & 0.0774 \\
185 & 0.1733 & 50 & 0.0752 \\
\hline
\end{tabular}


4

MEASUREMENT OF LASER EXTINCTION IN WARM FOG
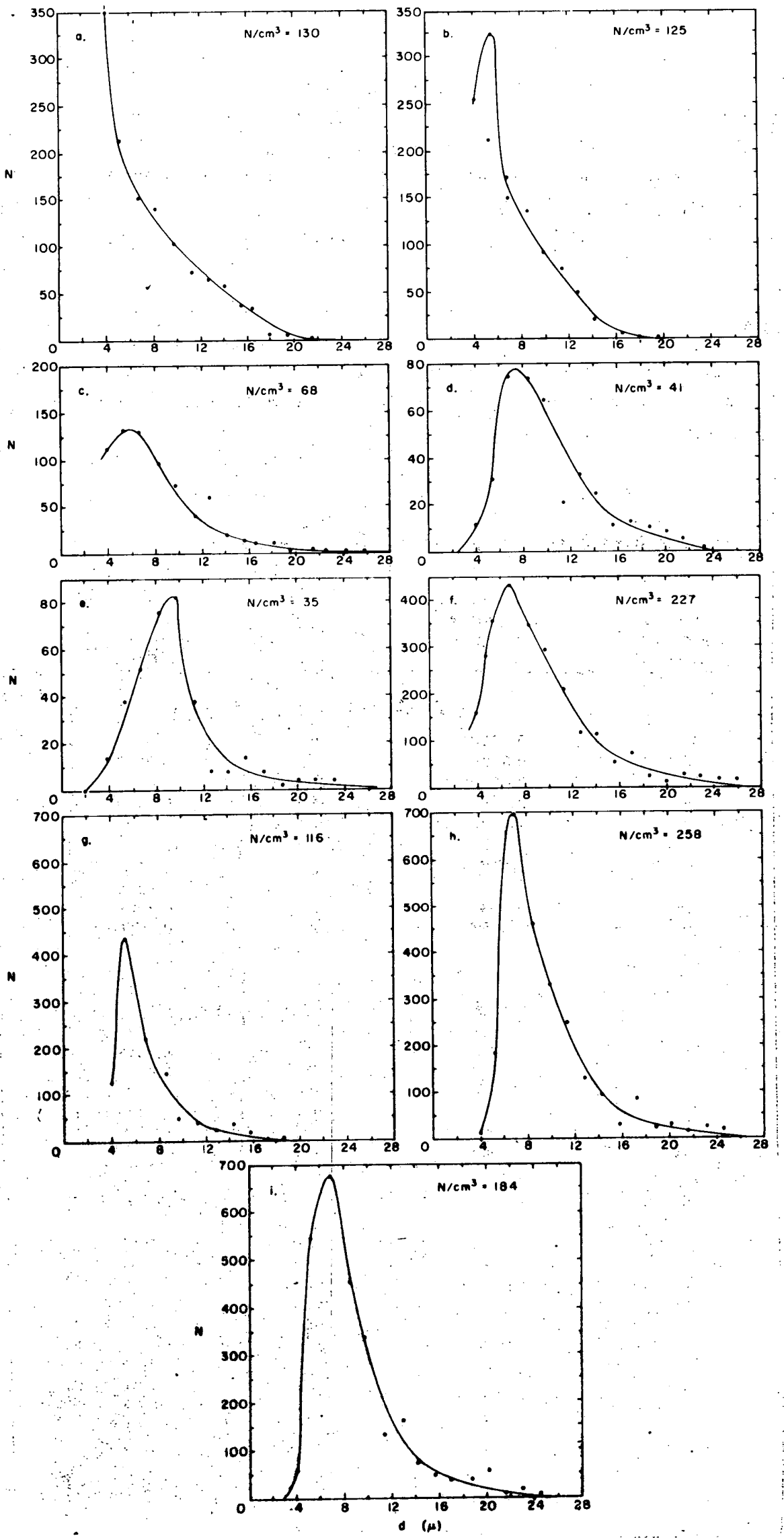

1

Figure 2. Particle concentration vs diameter at $-4^{\circ} \mathrm{C}$. 


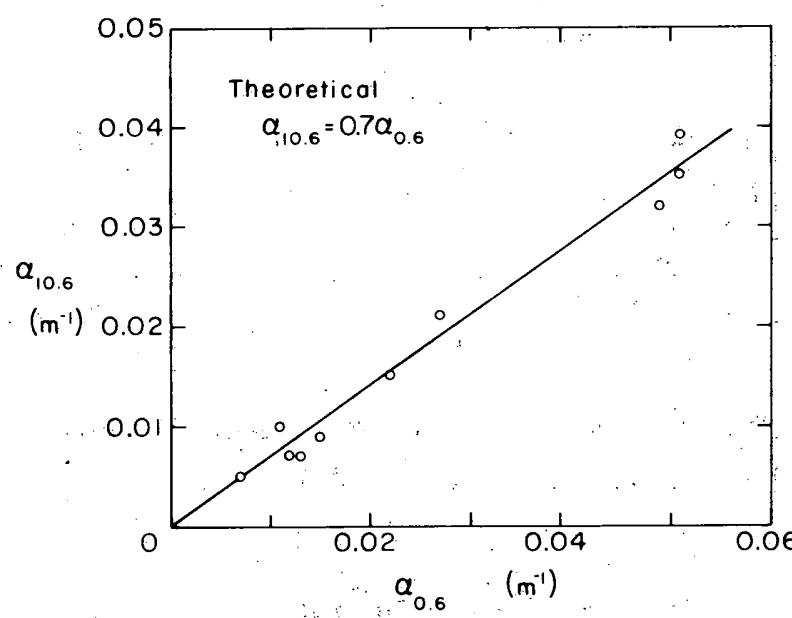

Figure 3. Theoretical extinction coefficient at $10.6 \mu$ versus theoretical coefficient at $0.6328 \mu$.

It is interesting to note that at $1.15 \mu$ the values of transmission are considerably lower (consequently with significantly higher extinction coefficients) than at 0.6328 and $10.6 \mu$. As mentioned before, a comparison of the theoretical values of the extinction efficiency (Fig. 1) would seem to indicate that throughout the computed particle spectra the extinction coefficients at 0.6328 and $1.15 \mu$ should have similar values. However, calculation of the theoretical extinction coefficient does not take into account the additional loss through water vapor which is present in the chamber during formation and dissipation of a fog. It is recognized that the wavelength of $1.15 \mu$ appears in a wing of the $1.125-\mu$ atmospheric $\mathrm{H}_{2} \mathrm{O}$ vapor band. In a review of the optical properties of ice and water, Irvine and Pollock ${ }^{2}$ indicate that the complex part of the index of refraction at $1.15 \mu$ exceeds that at $0.6328 \mu$ by approximately three orders of magnitude and that the absorption coefficient of water at $1.15 \mu$ is about 400 times greater than that at $0.6328 \mu$. These values could therefore explain why the experimental extinction coefficients are so much higher at $1.15 \mu$ than at $0.6328 \mu$. On the other hand experimental data obtained by Arnulf et al. ${ }^{1}$ do not seem to corroborate the data obtained in this experiment.

Since the radiation at $1.15 \mu$ is adversely affected by atmospheric water vapor, it is of interest to examine the relationship between the extinction coefficients at $0.6328 \mu$ and $10.6 \mu$. Figure 3 shows a plot of the theoretical extinction coefficients at $0.6328 \mu$ vs the coefficients at $10.6 \mu$. These coefficients were calculated using eq 2 and 4 and the measured particle size distributions. The slope of the curve indicates that the extinction coefficient at $10.6 \mu$ should be somewhat smaller than that at $0.6328 \mu$ or, conversely, that transmission through the fog should be somewhat higher at $10.6 \mu$. However, Figure 4 shows that the experimental results indicate that the extinction (or transmission) at $0.6328 \mu$ relative to that at $10.6 \mu$ becomes independent of particle concentration at approximately $200 \mathrm{~cm}^{-3}$. The slope of the linear portion of this curve indicates that the extinction coefficient at $0.6328 \mu$ is approximately equal to that at $10.6 \mu$. Since the optical depth $\tau$ is quite large at both wavelengths with the particle concentration in the neighborhood of $200 \mathrm{~cm}^{-3}$ it is quite probable that the full effects of multiple scattering are dominating the scattering process. (Table II gives transmission values and optical depths $\tau$ at 0.6328 and $10.6 \mu$.) Thus if only the linear portion of Figure 4 is taken into consideration the agreement with theory is not too bad. 


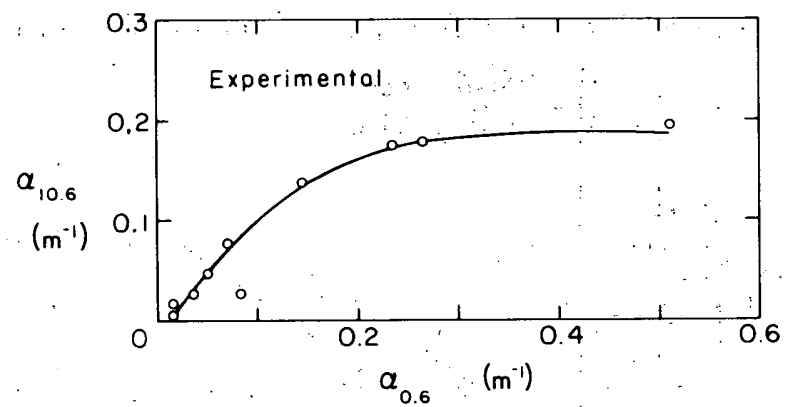

Figure 4. Experimental extinction coefficient at $10.6 \mu$ versus experimental coefficient at $0.6328 \mu$

Table II. Transmission values and corresponding optical depths at 0.6328 and $10.6 \mu$.

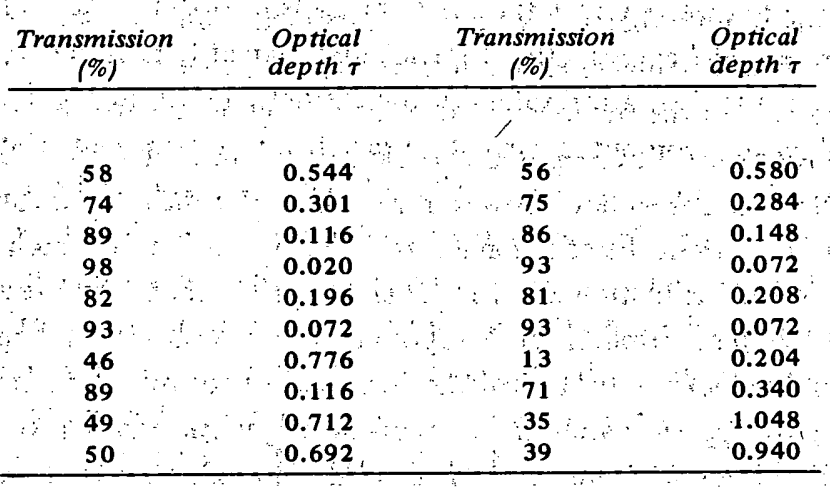

\section{Conclusions}

Experimental and theoretical data have been obtained on the simultaneous measurement of laser propagation through warm fog at $0.6328,1.15$ and $10.6 \mu$. It is theorized that due to high $\mathrm{H}_{2} \mathrm{O}$ vapor concentrations in the chamber, propagation at $1.15 \mu$ was severely reduced. This can be somewhat confirmed by the data in Figure 1, which indicate that for equal particle concentration the extinction coefficient should be approximately equal whether the particle spectrum peak is found at 7 or $12 \mu$. The $7-\mu$ peak was measured for three concentrations during this experiment (warm fog) while the $12-\mu$ peak was measured for approximately the same three concentrations (ice fog) and reported in ref. 3. The significant difference during these two experiments was that the ice fog propagation measurements were conducted at $-43^{\circ} \mathrm{C}$, which would tend to freeze out most of the water vapor, while the warm fog measurements were made at $-4^{\circ} \mathrm{C}$ and hence a larger amount of $\mathrm{H}_{2} \mathrm{O}$ vapor would be present in the fog chamber. This is illustrated dramatically when a comparison is made of the transmission values in Table III in ref. 3 and Table la in this report.

Theoretical calculations seem to indicate that the extinction coefficient at $10.6 \mu$ is somewhat smaller than that at $0.6328 \mu$ and thus should favor this wavelength if propagation through warm fog is the major concern of a design engineer. However, experimental data seem to contradict the theoretical calculations in that these data show virtually no difference between the extinction 
coefficients at these two wavelengths for moderate fog concentrations, while for extremely large concentrations $\alpha_{10.6}$ assumes a constant value of approximately 0.2 while $\alpha_{0.6328}$ increases indefinitely.

\section{Literature cited}

1. Arnulf, A., J. Bricard, E. Curé and C. Véret (1957) Transmission by haze and fog in the spectral region 0.35 to 10 microns. Journal of the Optical Society of America, vol. 47, p. 491-498.

2. Irvine, W.M. and J.B. Pollock (1968) Infrared optical properties of water and ice spheres. Icarus, vol. 8, p. 324-360.

3. Munis, R.H. and A. Delaney (1972) Measurements of laser extinction in ice fog. U.S. Army Cold Regions Research and Engineering Laboratory Research Report 302. 